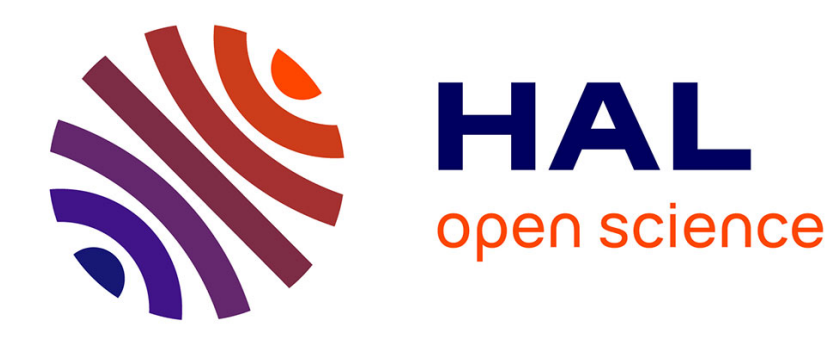

\title{
Voltage controlled metamaterial
}

Olivier Reynet, Acher Olivier

\section{- To cite this version:}

Olivier Reynet, Acher Olivier. Voltage controlled metamaterial. Applied Physics Letters, 2004, 84, pp.1198. hal-00911246

\section{HAL Id: hal-00911246 https://hal.science/hal-00911246}

Submitted on 29 Nov 2013

HAL is a multi-disciplinary open access archive for the deposit and dissemination of scientific research documents, whether they are published or not. The documents may come from teaching and research institutions in France or abroad, or from public or private research centers.
L'archive ouverte pluridisciplinaire HAL, est destinée au dépôt et à la diffusion de documents scientifiques de niveau recherche, publiés ou non, émanant des établissements d'enseignement et de recherche français ou étrangers, des laboratoires publics ou privés. 


\title{
Voltage controlled metamaterial
}

\author{
O. Reynet ${ }^{\mathrm{a})}$ and O. Acher ${ }^{\mathrm{b})}$ \\ Laboratoire Matériaux Magnétiques et Hyperfréquences, CEA Le Ripault, BP 16, 37260 Monts, France
}

(Received 19 September 2003; accepted 18 December 2003)

\begin{abstract}
A tunable metamaterial based on conducting coils loaded by an electronic circuit containing a varicap diode has been demonstrated. The agility exceeds one octave inside the $100-500 \mathrm{MHz}$ range. Permeability levels are significantly increased when the loop is loaded by a proper magnetic material. A model gives a good desription of the observed permeability spectra. (C) 2004 American Institute of Physics. [DOI: 10.1063/1.1646731]
\end{abstract}

The possibility to design structured composites with negative refractive index has been demonstrated. ${ }^{1}$ These metamaterials offer electromagnetic properties which are not present in nature. ${ }^{2,3}$ It is attracting to design tunable metamaterials. In a prior paper, ${ }^{4}$ we demonstrated the possibility to tune the permittivity of a lattice of magnetic wires. Active negative permeability inclusions have been considered theoretically, ${ }^{5}$ but to our knowledge, experimental investigations on such active or tunable elements are still lacking. Several types of negative permeability inclusions have been considered up to now, such as coupled-ring resonators, ${ }^{6}$ resonant coils, ${ }^{7}$ and lumped-element electronic circuits. ${ }^{8}$ In this letter, we report experimental results on tunable metamaterials built from conducting coils and electronic circuits. Different type of loops, some with a magnetic core, are investigated. The observations are compared to a model detailed elsewhere.

We build resonant permeability inclusions by connecting two types of coils to a capacitive tunable electronic load. The coils were made of a $200-\mu \mathrm{m}$-diam $\mathrm{Cu}$ wire wound into a 4-mm-diam helix. A two turns coil with an inductance of 39 $\mathrm{nH}$ and a five turns coil with an inductance of $107 \mathrm{nH}$ were used. A core of magnetic material with a permeability of $\mu$ $=2-j 0.1$ up to $500 \mathrm{MHz}$ could be inserted in the two turns coil, which changed its inductance value to $78 \mathrm{nH}$ and added small losses. The tunable capacitive load consists in a varactor diode in series with a $150 \mathrm{pF}$ capacitor. The bias voltage is applied to the diode through proper choke inductors to protect the voltage supply (see Fig. 1). The varactor has an hyperabrupt behavior, and can be tuned from 15 to $2 \mathrm{pF}$ when biased in the 1-20 V range. The permeability of a device made of one tunable element is measured using a shorted APL40 coaxial line connected to a network analyzer. The coil is located inside the coaxial line, in front of the short circuit, with its axis parallel to the microwave magnetic field. The tunable capacitive load is placed outside the line, behind the short circuit, and is connected to the coil by two small via through the short circuit (see Fig. 1). The normalized impedance of the device $z_{c c}=Z / Z_{0}$, where $Z_{0}$ is characteristic impedance of the line, is measured using the network analyzer and the shorted line. Its effective permeability is deduced from the first-order relation:

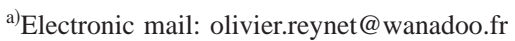

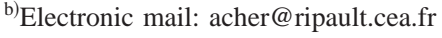

$$
\mu=-j c_{0} z_{c c} / 2 \pi e f
$$

where $c_{0}$ is the speed of light in vacuum, $f$ the frequency, and $e$ the thickness of the sample corresponding to the diameter of the coil. This approximation holds provided the thickness $e$ of the device is much smaller than the wavelength inside the device.

The complex permeability of the sample with the two turns loop is represented in Fig. 2, for a bias voltage ranging from 0.5 to $20 \mathrm{~V}$. A resonant permeability is observed, with a resonance frequency ranging from 200 up to $490 \mathrm{MHz}$ depending on the capacity of the load. The agility of the system exceeds one octave. The loss peak bandwidth $\Delta f$, where $\Delta f=f_{2}-f_{1}$ and $f_{1}$ and $f_{2}$ are the frequencies for which $\mu^{\prime \prime}\left(f_{1}\right)=\mu^{\prime \prime}\left(f_{2}\right)=\frac{1}{2} \mu_{\max }^{\prime \prime}$, ranges from 5 to $10 \mathrm{MHz}$. Using the five turns loop, a similar resonant behavior is observed (see Fig. 3). The resonance frequency can be tuned from 130 to $385 \mathrm{MHz}$, still achieving more than one octave agility. The loss bandwidth $\Delta f$ is about $8 \mathrm{MHz}$. Permeability levels associated with this larger loop are higher, and negative permeability levels are observed. When a piece of magnetic material is inserted in the coil (see Fig. 4), still higher permeability levels are observed. The agility range is hardly affected. The permeability loss peaks $\Delta f$ broadens significantly to $15 \mathrm{MHz}$ when the resonance is tuned at $140 \mathrm{MHz}$, and to $30 \mathrm{MHz}$ when it is tuned at $360 \mathrm{MHz}$.

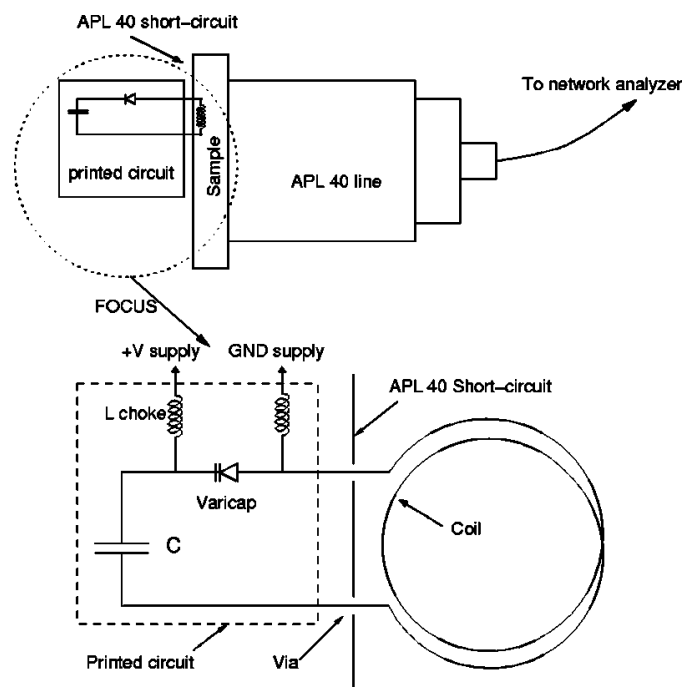

FIG. 1. APL 40 characterization device description and focus on the load electronic circuit of the loop. 

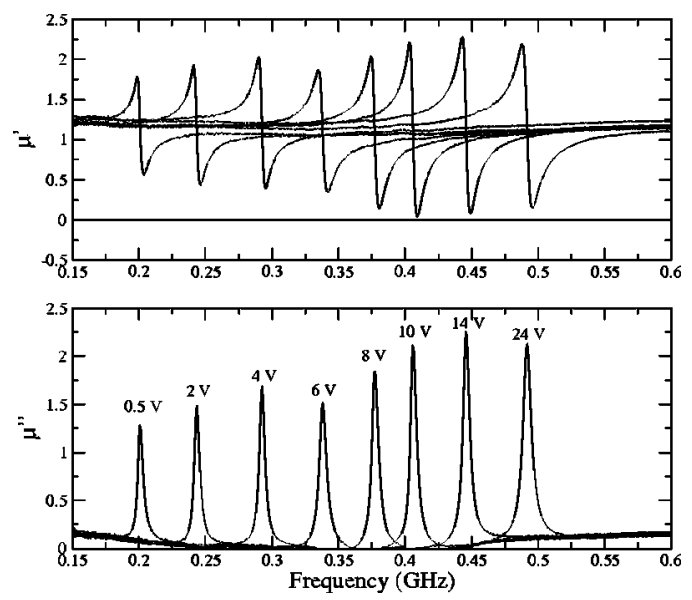

FIG. 2. Artificial permeability of the sample with a two turns coil and varicap diode. Bias voltage is indicated above each curve.

The results reported here can be accounted for using the appropriate definition of the permeability for a composite material containing current loops. ${ }^{9}$ In this case, the permeability of the device is given by

$$
\mu_{0} \mu_{\mathrm{eff}}=\langle B\rangle_{V} / H
$$

where $\mu_{0}$ is the permeability of the vacuum, $\langle B\rangle_{V}$ is the volume average in the composite, and $H$ the field outside the conducting loops. In the samples under consideration, large currents flow through the coils at the resonance frequencies of the circuit. These currents result in a large $B$ inside the coil, which accounts for the permeability levels significantly different from unity close to the resonance. Since the unwinded length of the coils is much smaller than the wavelength, the current is nearly constant along the coil. The expression of the current as a function of the impedance of the load and of the loop is rather straightforward. Using this approach, we have been able to show that ${ }^{10}$

$$
\mu_{\mathrm{eff}}=1-\alpha+\alpha \mu_{c} \frac{Z_{c}}{Z_{b}+Z_{c}} .
$$

where $Z_{b}$ stands for the impedance of the coil, $Z_{c}$ the impedance of the load, and $\mu_{c}$ the permeability of the core in the loop. $\alpha$ corresponds to the volume fraction of inclusions within the composite. The permeability computed from the
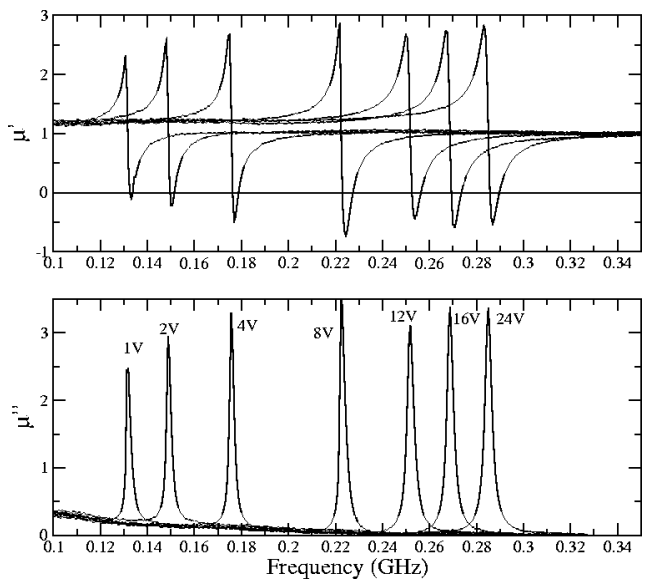

FIG. 3. Artificial permeability of the sample with a five turns coil and varicap diode. Bias voltage is indicated above each curve. varicap diode. Bias voltage is indicated above each curve.
Downloaded 19 Dec 2007 to 132.165 .42 .23 . Redistribution subject to AIP
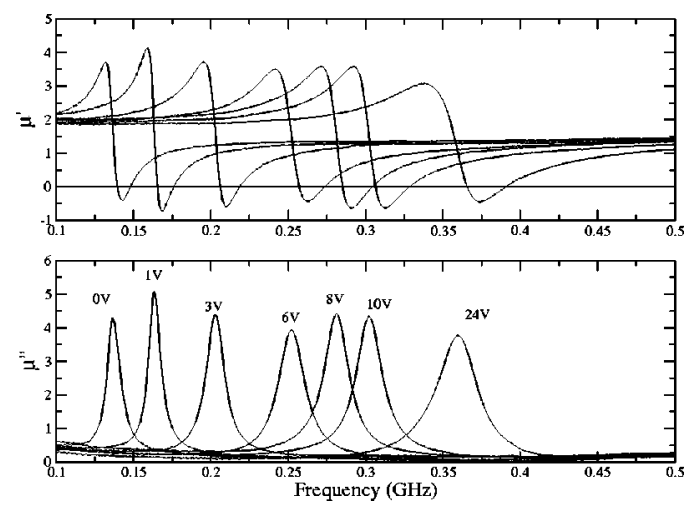

FIG. 4. Artificial permeability of the sample with a two turns coil, varicap diode, and magnetic composite in the loop. Bias voltage is indicated above each curve.

experimental values of $Z_{b}$ and $Z_{c}$ is in excellent agreement with our observations, as evidenced in one example represented in Fig. 5. Besides, it is straightforward to see that for a coil with $Z_{b}=j L \omega$, a capacitive load with $Z_{c}=1 / j C \omega$, the resonance frequency $F_{r}$ is given by

$$
F_{r}=1 /(2 \pi \sqrt{L C}) .
$$

Figure 6 shows that this relation accounts for the observed tunability. The control of the permeability of a metamaterial through a bias voltage is an attractive feature, compared to the usual way to tune the permeability of a magnetic materials through an external magnetic field. ${ }^{11}$ Bias magnetic fields may be power consuming and bulky to implement. Besides, the model reported here allows the engineering of the permeability response through a proper conception of the load circuit.

One may object that the permeability levels reported here are moderate, and that negative permeability is achieved only over a very limited bandwidth. It is clear that the devices investigated here are very diluted. The coils have a foot print of 10 and $15 \mathrm{~mm}^{2}$, which corresponds, respectively, to $1.1 \%$ and $1.6 \%$ of the $907 \mathrm{~mm}^{2}$ overall cross section of the coaxial line. Increasing the volume fraction $\alpha$ of the coils by having more elements per area is a straightforward way to
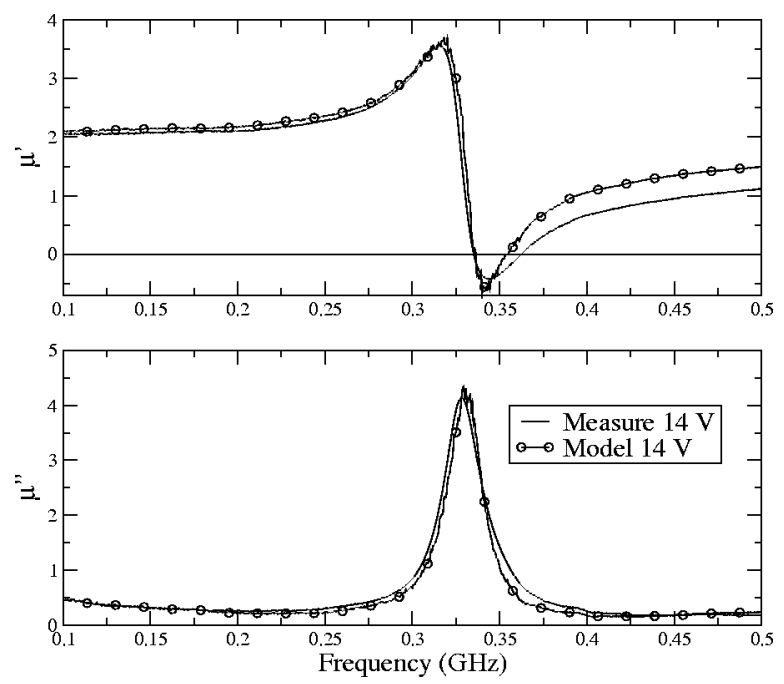

FIG. 5. Measurement and modelization of artificial permeability of the sample with a two turns coil, varicap diode, and magnetic composite in the 


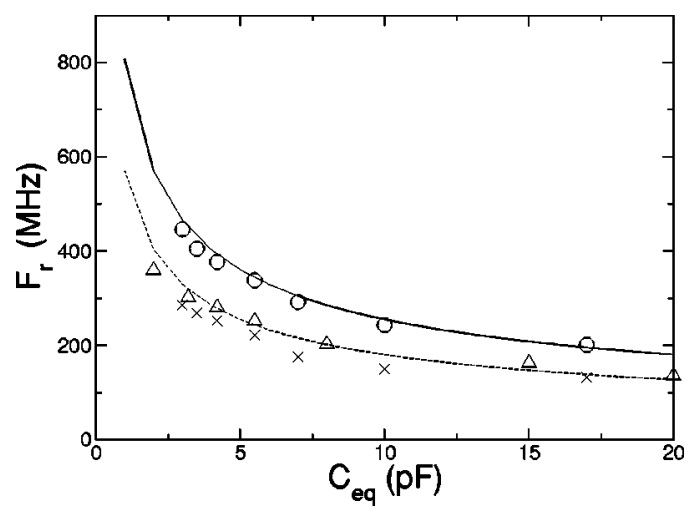

FIG. 6. Resonance frequency of the metamaterial vs the total capacitance $C_{\mathrm{eq}}(150 \mathrm{pF}$ and the varactor diode). Experimental observation corresponding to the two turns coil (circles), two turns coil loaded with the magnetic core (triangles), and five turns coils $(\times)$. Solid lines correspond to the model for the first two cases.

increase the permeability levels, as shown by Eq. (3). The use of high permeability cores is also favorable, as demonstrated by Fig. 4 and supported by Eq. (3). Another objection may arise from possible chiral effects in the structures we used. If these effects are not desired, the use of double he- lixes with opposite rotations has been shown to prevent the occurrence of unwanted chiral effects. ${ }^{7}$ As a summary, it is shown that metamaterials with tunable permeability can made from simple capacitive circuits. This offers significant possibilities for engineering the response of metamaterials, and more generally magnetic microwave materials.

${ }^{1}$ J. Pendry, Phys. World 14, 47 (2001).

${ }^{2}$ R. Shelby, D. Smith, S. Nemat-Nasser, and S. Schultz, Appl. Phys. Lett. 78, 489 (2001).

${ }^{3}$ D. Smith, D. Schurig, M. Rosenbluth, and S. Schultz, Appl. Phys. Lett. 82, 1506 (2003).

${ }^{4}$ O. Reynet, A.-L. Adenot, S. Deprot, O. Acher, and M. Latrach, Phys. Rev. B 66, 094412 (2002).

${ }^{5}$ S. Tretyakov, Microwave Opt. Technol. Lett. 31, 163 (2001).

${ }^{6}$ J. Pendry, A. Holden, D. Robbins, and W. Stewart, IEEE Trans. Microwave Theory Tech. 47, 2075 (1999).

${ }^{7}$ A. Lagarkov, V. Semenenko, V. Kisel, and V. Chistyaev, J. Magn. Magn. Mater. 258-259, 161 (2003).

${ }^{8}$ G. Eleftheriades, A. Iyer, and P. Kremer, IEEE Trans. Microwave Theory Tech. 50, 2702 (2002).

${ }^{9}$ O. Acher, A. Adenot, and F. Duverger, Phys. Rev. B 62, 13748 (2000).

${ }^{10} \mathrm{O}$. Reynet, these de doctorat, Universite de Bretagne Occidentale, France, 2003.

${ }^{11}$ O. Acher, P. Baclet, G. Perrin, O. Roblin, and P. L. Gourrirec, J. Magn. Magn. Mater. 157-158, 480 (1996). 